\title{
Ecological aspects of the education and resilience of preschool teachers
}

\author{
Olga Bagadaeva $^{1}$, Marina Golubchikova ${ }^{1,2}$, Elena Kamenskaya $^{3}$, and Mariam Arpentieva ${ }^{3 *}$ \\ ${ }^{1}$ Pedagogy Institute, Irkutsk State University, Psychology and Pedagogy of Preschool Education \\ Department, Department of Education, 664011, Irkutsk, Russian Federation \\ ${ }^{2}$ Irkutsk State Medical Academy of Postgraduate Education - Branch of the Russian Medical \\ Academy of Continuing Professional Education of the Ministry of Healthcare of the Russian \\ Federation, Department of Pedagogical and Information Technologies, 664003, Irkutsk, Russian \\ Federation \\ ${ }^{3}$ Institute of Management in the Economic, Environmental and Social Systems of the Southern \\ Federal University, Department of Ecology and Life Safety, 347928, Taganrog, Russian Federation
}

\begin{abstract}
An individual acquires environmental knowledge and skills (competencies) in several ways. In addition to direct training within the framework of special courses, the ecology of everyday interactions and human behavior is of great importance, especially in the early stages of development, starting with preschool age. The ecology of pedagogical interaction as a system of environmentally literate relationships between educational subjects includes a number of indicators, including those related to the formation of the personal prerequisites for such interaction. One of them is the teacher's resistance to stress, which he forms and develops also in his wards. The aim of the study is to analyze the resistance to stress of a teacher as a factor in the ecology of educational relations and interactions. The profession of a teacher in a preschool institution is highly and diversely stressful, requiring self-regulation (self-management), due to a significant psychological load that exceeds that of many other specialists. Stress resistance of a teacher of a preschool educational institution plays an important role in building and improving didactic relations, as well as the formation and development of environmental literacy and human culture.
\end{abstract}

\section{Introduction}

An individual acquires environmental knowledge and skills (competencies) in several ways. In addition to direct training within the framework of special courses, the ecology of everyday interactions and human behavior is of great importance, especially in the early stages of development, starting with preschool age. Ecological psychology of education, psychoecology of education, pedagogical ecology (A.M. Adamenko, I. Altman, R. Barker, E. Hall, M. Heidmets, T. Högerstrand, J. Gibson, V.V. Glebov, A.A. Kalmykov, K. Kraik, J. Kruusvall, K. Levin, V.I. Medvedev, G. Murray, W. Michel, T. Niit, V.I. Panov, E.A. Sterligova, I.V. Smirnov, V.I. Vernadskiy, R. Sommer, D. Stokols) as sections of ecology

* Corresponding author: mariam rav@mail.ru 
study the ecological properties of the educational environment, the influence of the psychodidactic characteristics of this environment on actions and relationships, on behavior and communication, on personal, interpersonal and professional formation and development of an individual and society. The ecology of pedagogical interaction as a system of environmentally literate relationships between educational subjects includes a number of indicators, including those related to the formation of the personal prerequisites for such interaction. One of them is the teacher's resistance to stress, which he forms and develops also in his wards. Stress resistance of a teacher of a preschool educational institution plays an important role in building and improving didactic relations, as well as the formation and development of environmental literacy and human culture.

Within the framework of the concept of the ecology of the educational process, we can talk about pedagogical ecology, one of the principles of which is set by the law of tolerance (W. Shelford): the limiting factor for the prosperity of an organism can be at least or a maximum of environmental impact, the range between which limits the value of endurance (tolerance) of the organism to this factor. The law of optimum is no less important: any environmental factor has certain limits, positive effect on living organisms. In education, it is important that the factors contributing to the normal development of a person are maximized and the factors that hinder him are minimized. With regard to stressful influences, this means maximizing the competencies and activity of teachers in relation to the prevention and correction of distress states in themselves and preschoolers, schoolchildren and students, as well as minimizing the influences and states that increase conflict and frustration, stresses and their consequences. "proportion" here is defined in different ways, for example, using the law (rule) "10\% R. Lindemann": the average maximum transition from one trophic level of the ecological pyramid to another $10 \%$ of energy (or matter in energy terms), as a rule, does not leads to adverse consequences for the ecosystem and the trophic level losing energy. This rule is closely related to the idea of the importance of a gradual and conscious educational and training impact, prompting a person to move to higher levels of development, without destroying the foundations of his / her existence. Within the framework of ecology, it is noted that the energy received by the community and assimilated by producers is dissipated or, together with their biomass, is transferred to consumers, with a drop in the flow at each trophic level. This suggests that the efforts of a teacher striving for the development of his students can be very significant, and their final expression - almost imperceptible, hidden: whole systems of pedagogical influences and measures can lead to "point" changes in the higher levels of organization, for example, the formation in a child preschool age, the ability and readiness of planning and prospective understanding of oneself and the world, for voluntary / volitional behavior, for decentration and dialogue, the formation and consolidation of moral norms and values, etc. Also, based on the A. Lotka and V. Volterra equation and the exclusion law of G.F. Gause, biological species cannot exist if they occupy the same ecological niche. If two competing species coexist in a stable environment, this occurs as a result of niche differentiation. It is important, therefore, that the children in the preschool educational institution are approximately the same in development, so that the teacher can pay due attention to everyone, and not to the species that will be more capable of "survival": in the upbringing and training of an individual, the category of survival has a special meaning associated with the need the formation of a person's competencies that allow him / her to live with dignity, independently, in peace with himself / herself and with other people, etc.

The modern system of education and upbringing all over the world is going through large-scale changes that cause an increase and elderly people) [1-3]. Due to the numerous transformations and reforms in education, culture, and life of the country as a whole, sometimes reminiscent of a "snowball" or an avalanche, representatives of the teaching 
profession find themselves in high-risk zones, zones of increased and sustained danger of educational and related relations.

The passage of a teacher as a person, partner and professional of certain stressful situations inevitably sometimes has a very intense impact on the life and condition of the teacher, on his health and personality traits, on relations with others and on the performance of his duties and general professional traits. Of course, many stresses in the work of a professional can be attributed to professional, closely related to the specifics, processes and results of professional work. But many situations and stresses can be attributed to universal, including general cultural, macrosocial: the teacher does not live in a vacuum, but, most often, "at the forefront" of sociocultural transformations. Beginning as changes in the field of education, these transformations penetrate and become norms in other spheres of everyday life. M.R. Arpentieva and colleagues have shown that professional stress among teachers is often associated with "numerous educational reforms and toughening requirements for teachers" and other teachers. Modern teachers in completely different ways and to varying degrees withstand the significant professional and accompanying professional loads that fall on their lot due to various reasons $[4,5]$. They almost constantly go through situations oversaturated with conflicts, difficulties, stresses, often leading to crises of their personal, interpersonal and professional functioning. Destructive and negative psychological states and the dysfunctional traits of the teacher as a person, partner and professional at each stage of the educational system that they form sharply reduce the quality of the effectiveness and productivity of the upbringing and education of children, adolescents, youths, adults, and the elderly. They, as a rule, significantly increase and normalize conflict and even crisis in teachers' relations with their pupils, parents, colleagues, with society as a whole, destroy psychological and somatic health, professional identity and competence, life satisfaction and psychological well-being [6-8].

Recently, the phenomena of professional burnout and didactogenies of pedagogical workers, cases of personal, interpersonal and professional deformations of employees of the entire education system, passivity and rejection of innovations, object-oriented attitude towards students, bullying and other forms of conflict and alienated behaviour and relations in education have become more and more common [2, 3, 9, 10]. Communities and states noticeably increase claims, critical and, at the same time, indifference to teachers, to the processes and results of pedagogical activity, to education in general. On the wave doctrines of "educational services", the role of the teacher is levelled to an employee who, due to production needs, can be directed to perform any educational service or other activity that is not part of his professional duties. Regarding the scientific and methodological activities of the teacher, they are perceived as a developer of educational documentation, for which (as well as for scientific and other research and development in general), many teachers not only have no rights, but, by default, are deprived of authorship: everything that the teacher produces while working in an educational organization, it is recognized as the property of the organization, which it is free to dispose of (sell and acquire) at its own discretion, bypassing any agreements and deductions to the teacher. Thus, the teacher is losing his position both as a specialist in the organization and implementation of cultural transmission, and as an author of developments and documentation that underlie the so-called "educational" services. The accumulated pedagogical experience and achievements in this area do not have sufficient recognition from colleagues, since they are demonstrated in a situation of active competition for the accrual of incentive payments or in an impersonal form of a model passport or other certification documentation for obtaining a qualification category. 


\section{Materials and methods}

The purpose of the research is to analyze the problems of stress resistance of preschool teachers.

The research method is a theoretical analysis of the problems of stress resistance of preschool teachers. Stress resistance of the teacher is considered as a component of the psychological and pedagogical safety of education. It is also seen as a model of response that the teacher passes on to the preschooler, which guarantees environmental friendliness.

\section{Research results and its discussion}

The problem of stress resistance of a teacher is in great demand and studied both in Russia and abroad. On the one hand, it is traditional, but on the other hand, it is very rich. The conceptual field of research in this area concentrates on several basic concepts, including the concept-forming concept of stress by H.H.B. Selye and his school [11]. Resistance to stress as the ability and readiness to withstand stress and its negative consequences, as well as prevent stress and minimize the causes that lead to them, is one of the traditional concepts with numerous interpretations. The areas of research on human stress resistance are most richly represented by the areas of educational and helping activities, as well as activities in extreme conditions (education, social and psychological assistance, healthcare, law enforcement, etc.). In the field of education, the problem of resistance to stress covers private and integrative, including comparative studies of stress resistance of subjects of education of different levels and different types, including stress resistance among preschool teachers. The conceptual field of stress resistance research includes such concepts as resilience and life-affirmation, self-realization and self-actualization, selfefficacy and social efficiency, psychological well-being and satisfaction, psychological safety, professional acme and professionalism, creativity and dialogue, self-regulation and self-management, will and awareness, reflection and critical thinking that allows you to comprehend situations in a flexible, multidimensional, multilevel, etc., optimism and positive redefinition of difficult life situations and traumatic life experience.

The opposite pole to stress resistance is represented by the concepts of psychological danger, dissatisfaction and distress, ineffectiveness and refusal to develop, anxiety and depression, deprofessionalization and routinization, monologue and numerous deformations of the personal, interpersonal and professional type, including psychological burnout, demotivation and desacralization, transgression, ressentiment, post-traumatic stress disorders, including didactogenic disorders (pediogenya, mathetogenya, eductogenya), conflicts and bullying, the concept of monologue and reproduction, resistance and stress of innovation, lack of will and learned helplessness, a-reflexivity, cognitive negative simplicity (flattening and stereotypism). The multitude of concepts, therefore, indicates that the problem, being largely successfully and long ago developed, is still far from being resolved: new challenges and crises of social relations, including the crises of education itself in the modern world, give rise to new difficulties. new phenomena that either increase or decrease an individual's resistance to stress.

Many studies of human resistance to stress and trauma abroad are devoted to the problems of psychological well-being of an individual as a person, a partner and a professional: many of them have been carried out by N. Bradbum, E. Diener, R. Ryan, L. Desi, S. Riff and A. Waterman and researchers developing them, resource, approach. Psychological well-being is one of the important resources and indicators of human stress resistance [12]. The psychological resources of a person, providing psychological wellbeing, happiness, and other analogist of the state of satisfaction with oneself and the world, are fully described in the works of D. Kahneman, R. Lazarus, S. Folkman, J. Lowinger, C. 
Rogers, V. Frankl, E. Fromm, S. Hobfall, A. Maslow, A. Baker, E. Demerouti. The resource approach to self-regulation of mental states and activity is addressed to the idea of conscious ("cognitive") management of the distribution of mental resources, building relationships, as a process and result of successful solution of professional tasks [13].

In Russia, in addition to the resource approach, the activity-procedural approaches are also actively developing [14-16]. Researchers draw attention to the fact that the interaction of a person and the world is dialogical and unpredictable, resources and associated personal, interpersonal and professional patterns (stereotypes) can sometimes interfere with a person, and not help him cope with a problem. Therefore, an important point is the ability and willingness of a person to change, to let go of traumatic, stressful experiences and related ideas and experiences (disorientation and uncertainty, fear of lack, anger / aggression, guilt / blame, etc.). The most important thing here is motivation for changes, including professional ones, for career and professional growth and competence. It is motivation and its value-semantic foundations that suffer in the first place, when they talk about psychological burnout and other violations of teachers, up to deformations and didactogenies.

So, S. B. Velichkovskaya, as before, V. Frankl, notes that the ability to overcome stress increases with strong motivation, and its absence reduces stress resistance and resilience of an individual. Motivational involvement in activities, value meaningfulness of work, manifested in satisfaction with one's work, oneself, life (that is, in the psychological wellbeing of a specialist) significantly transforms the comprehension and coping with stress, changing the direction and results of work with a stressful situation and the experience of coping with it. Dissatisfaction with activity, meaninglessness of activity, deformed motivational structure of activity leads to the fact that a person falls into a group of increased risk of occurrence and development of (di)stress. Scientists study stress resistance in the context of adaptation to professional activity, linking it with the productive orientation (focus on development) of the teacher's psychological adaptation [17].

Here, the role of meaning-life orientations, life-affirmation as the basis of an individual's vitality is especially great $[2,3]$. Researchers also note the role of special training activities for the prevention and correction of distress and activities for the application of methods of stress management and stress management assigned in the course of education and upbringing, including family, preschool, school, and university. Thus, they note the importance of implementing a health-preserving, psychologically safe, selfhealing, psychoecological approach to stressful situations, the importance of reflecting on what is happening, helping to preserve and restore the integrity (self) of an individual as a person, partner and professional [4]. Self-healing / self-healing techniques (self-clarification and self-harmonization) should be aimed at activating and building up a person's reserves, his health and stress resistance, and not just "waste". Stress is a situation that challenges a person: a challenge associated with inevitable losses, but also inevitable gains. The result of stress is the choice of the person himself, including the choice of psychological well-being and distress [3, 18, 19].

Stress is often defined as a protective reaction of the body aimed at overcoming life situations, and as an effect that causes the body's need for protection [2, 17]. Stress is associated with the experience of a situation of danger or, at least, a situation that requires orientation: activating orientational activity, the instinct "what is it?" This suggests that the concept of "protection" does not cover the whole concept of stress and its functions, as well as that stress is needed by the body, it, at least, promotes the activation of "taste for life", motivation of vital activity, directs the search for a person in changes in the inner and outer world. This is eustress or positive stress. Without stress, a person falls into states described as "sensory" and other types of deprivation, isolation from oneself, others, work, life, and leading to the destruction of an individual as a person, a partner and a professional. Thus, 
stress has a constructive function: it is designed to increase the resistance of the human mind and body to unfavorable factors, to expand its functionality and vitality. Constructive stress eustress also mobilizes human resources, "awakens" dormant abilities and functions of a person, and helps to adapt to new environments and experiences. However, without control, including without prevention and correction, stress can become destructive: destructive stress - distress is associated with intense internal (re) stress, often chronic, causing depression (will, consciousness, humanity).

The concept of distress is associated with states of suffering, grief, exhaustion [20, 21]. Eustress becomes uncomfortable if a person is passive, lazy, procrastinates or "indulges" (looking for excuses for inaction, failure, etc.): comfort is opposite to the state of seeking and achieving (personal, interpersonal and professional) goals and their successful achievement. Self-efficacy and social efficiency of initiating, resolving conflicts, establishing agreements, etc., are the result and condition of eustress. But even eustress, with its constant, excessive, indefinite development, can turn into distress: general depression and dissatisfaction with life, a lack of life resources (vitality, supportive relationships and meaningful goals), and the presence of pressure from circumstances and specific people and organizations dispose of distress.

Common causes of distress and its persistence and aggravation include the following:

1. A person lives in poverty, does not have a job, family, housing, is driven by unresolved difficult life circumstances, constantly suffers defeats and humiliations (macrosocial and microsocial conflicts and crises), he is a victim of more or less persistent and conscious persecution by one or another people, groups, society;

2. A person at the moment and in a given place of his life cannot correspond to his own ideals, the ideals of loved ones or society, cannot find and realize his vocation, destiny. His life is sharply limited, poor, monotonous, or, on the contrary, oversaturated with events that have a traumatic effect (a state of bullying and bullying, a state of isolation and stigmatization, conditions associated with the experience of traumatization as a result of an emergency, etc.);

3. psychologically dangerous organizational climate (organizational culture), excessively stressful and long (irregular) work, labor rush jobs, including those related to innovations and transformations of labor, conflicts at work, etc .;

4. a person is prone to frustrations, seeks to see negative in everything, overestimate and take a hard time losing and - the habit of reveling in his depression, not wanting and not being able, for one reason or another, to say goodbye to her, to let her go and the injuries that caused it. Typical are constant accusations of others and self-accusations, reproaches to oneself and others that something has not been achieved or missed ("everything is not the way I want", dissatisfaction with life and dissatisfaction with self),

As a rule, signs of distress such as low energy potential, including physical weakness, mental inactivity, bad and / or unstable mood / mood, can in themselves also become sources of stress, perpetuate it, or induce a person to escalate stress and escalate violence. over oneself and the world: a person begins to suffer because he suffers, etc. Here, the connection between the resistance of teachers to stress and the tendency to reproduction of accumulated experiences is noted as an attempt to "work off", rethink the traumatic experience

At the same time, a number of other important points can be identified that characterize the state of research in the area we have chosen. Thus, in one of the large recent Russian reviews prepared by A.A. Khadartsev and colleagues [22] note that the work of a teacher is one of the most psychologically intense forms of professional work: through the stress of the processes of psychological adaptation, various disorders of the mental and physical health of teachers, psychosomatic diseases with rigidity, professional burnout, a high level of frustration, increased anxiety and anger, leading to professional deformations. Stress 
conditions the possibility of impulsive actions and a decrease in self-regulation. E.V. Barinova and many other researchers of the work of a teacher in the field of preschool education, talk about the profession of a teacher of a preschool institution as highly stressful, requiring developed knowledge and skills (competencies) in the field of selfregulation, self-control, due to a significant psychological load that exceeds that of many other specialists [23].

A.S. Balyk and I.P. Makarenko [24] analyze in sufficient detail the stress factors in the work of a preschool teacher, without highlighting, however, like many other scientists, narrowly specific ones that distinguish them from teachers of secondary and higher schools. In our study, we also focused on the most general points of stress resistance of a preschool teacher, analyzing which we can note that in work situations this teacher is faced with the need to harmonize the relationship between children and their parents and grandparents, as well as the relationship between the child's family members and the administration $\mathrm{He}$ is also faced with the problems of underestimating by students of their activities, which, due to its "elementary nature" or slow dynamics, is sometimes ignored. However, it is in preschool education that a child can and should get the opportunity to learn moral attitudes, value relationships, get acquainted with the concepts that are basic for the entire course of elementary and secondary school education, form readiness as a desire and desire, as well as the ability to study in primary and then secondary school and Further. This responsibility is very significant: the educator, in fact, lays the foundations for the ability and desire to learn, encourages parents to comprehend and take this or that position in relation to the child as a future student and even a professional. In general, his work as a "tuner" and "harmonizer" of educational and cognitive activity and positive socialization of a child in society as a whole is of great but consistently underestimated value. In "elite" preschool educational institutions, this problem is partially solved, but not always completely. Teachers, psychologists, defectologists, doctors, social workers are aware of the importance of the preschool stage of a child's development. But they paradoxically underestimate their role (the role of the preschool educator) in this development. At the same time, they act as a model of schoolchildren's behaviour, which forms behaviour patterns almost at a subconscious level.

This responsibility and its almost complete ignorance by researchers and the administration leads to the fact that the priority moments are the moments of self-reflection and responsible choice of the teacher, the moments of his self-regulation and management, allowing him to manage and, by controlling, change the attitude of the child and his family members towards himself / herself as the student and future professional, helping him to productively and effectively prepare the child for the next stage of socialization, the stage of school education. Therefore, the problem of psycho-prophylactics of stress and coping with stress in professional life (as well as outside of professional life) of a teacher of a preschool educational institution is very relevant: any violation in the personal, partner or professional sphere somehow penetrates and deforms the state of other areas.

The study of professional and other stresses, as well as stress resistance and, more broadly, the life resilience and life affirmation of the teacher, is necessary to prevent the occurrence and development of injuries, associated barriers, failures and other disturbances in the (life) activities of a preschool teacher, including avoiding decreased performance and vitality, the occurrence of professional deformities and professional burnout, as well as mental and psychosomatic disorders, often called didactogenies. In the modern education system (upbringing and training), there is a high level of conflict and stress in the educational environment, up to traumatisation and collapse of the personal, interpersonal and professional life of a person and a group. All this leads to the development and stability of stressful states and situations of (di)stress among teachers, which further affect their 
psychological and somatic health and the health of their wards (educated, trainees, students).

To ensure the social security of the subjects of education, the formation of their state of security in modern society, the teacher in the process of professional training should acquire stress resistance and such socially and professionally important qualities and competencies as knowledge and skills in the field of psychosomatic, social and moral selfregulation / self-regulation, reflexivity or "Critical thinking", stable spiritual, moral and value-semantic orientations, stable professional orientation and responsibility, knowledge and skills in the field of anticipation / planning and design, knowledge and skills in the field of managing interaction with other people and managing situations.

Achieving and strengthening stress resistance as an important professionally significant quality is based largely on developed universal (general cultural, key) competencies and the need for their development therefore serves as a condition for the preservation and development of the teacher's mental and somatic health, and, after that, the effectiveness of the entire educational system [11].

Stress resistance is formed and modelled through the creation and maintenance of a psychologically safe, comfortable working environment, the work of managers, psychologists and teachers themselves, etc. However, it is also associated with the internal circumstances and characteristics of a person: in addition to the macrosocial and microsocial external conditions for the emergence, development, comprehension and passage of a stressful situation and coping with stress, the internal conditions of these processes are extremely important. By themselves, stressful situations (and the very concept of stress) are so multifaceted and cognitively dependent on individual perception that, in fact, any change, and even the absence of change, can be considered stress. Stress as a response to a particular event or impact is subjective: the event is conceptualized and transformed as more or less significant, positive / negative or destructive / constructive.

The comprehension and passage of stress is influenced by the personality and interpersonal traits and competence of a person: his ability and readiness for self-regulation and self-government, as well as his readiness and ability to manage relationships with other (significant) people, holistic situations and even people themselves. R. Matush understands stress resistance as an axial property of an individual's ability to recover from deformations of the states and properties of an individual as a person, a partner and a professional arising in the process and as a result of exposure to stress / distress / traumatic factors and situations [26]. Resistance to stress is an important factor in the efficiency of work. A teacher with a high level of stress resistance perceives problem situations arising in professional activity not as threatening, stressful, but as situations that require resolution [27]. The comprehension and passage of stress is inevitably influenced by the professional culture and competence of a person. Professional competence is not so much a characteristic of the teacher's professional and related knowledge and skills. The implementation of competence at the level of self-regulation in its application, experience and knowledge demonstrates the true competence of the teacher. In this case, it acts as the leading component of pedagogical skill, reflecting the measure of the realization of a person's pedagogical abilities, associated with the measure of comprehending and mastering the methodology and content of education and training. It also determines the general interest and motivation of the professional, the specialist's desire to be creative and to master new facets of his profession, as well as to comply with the norms and traditions of pedagogy. It determines the harmony of self-realization and self-actualization of an individual as a person, partner and professional.

Resistance to stress is the most important internal factor of productive and effective coping with stress, associated with a number of points: 
1) with the general (macrosocial and microsocial) conditions of human life: supporting him or contributing to victimization, deformations, etc. This can include the general wellbeing of people's lives, the crisis or stability of their existence at one stage or another of life, the crisis and stability of the existence of the education system and the educational organization in which the teacher works, the features of family and clan relations and support systems such as "closest helpers" and organizations of "social protection of the population" [18, 24, 27];

2) with the wealth and awareness ("elaboration") of a person's life, including professional, experience: skills and knowledge in the field of understanding stress and coping with them, with the richness of the coping palette / coping register, that is, the presence and quality of active and passive, productive and destructive, mature and infantile strategies for coping, transforming a stressful situation and its experience: this moment is closely related to the quality of professional training and the teacher's experience, with his personal and interpersonal maturity. Thus, experienced teachers, using such behavioural strategies as positive re-evaluation and self-control, actively use humour in tense situations of professional activity. Young teachers characterized by such a behaviour strategy as escape - avoidance: they make efforts to avoid the problem; a stressful situation is perceived as a threat to their incompetence [28];

3 ) by the efforts and resources of a person, allowing him to search / find and apply / improve the methods and strategies of anticipation, projecting his activity in the direction of minimizing its stressfulness and psychological danger for himself and others, to apply individualized / individual, interpersonal / interpersonal and actually professionalized technologies of liberation and processing of traumatic, stressful experience, helping to use it for their development, and not destruction. This includes such important internal resources and conditions for human development that are associated with skills and knowledge (competencies) in the field of self-regulation and self-government, as well as the regulation and management of other people and their conditions. A teacher is a person who has professional experience, methods and competencies in the field of management and self-management of upbringing and training a person, including his upbringing and training in the field of coping with stress and stress prevention.

So, M.M. Kashapov notes the existence of a connection between the type of coping strategies and career and professional achievements, education level and professional experience / length of service of a person. He also revealed the connection between the over-situational problematization of current pedagogical problem situations and the success of labour, showed the impact and interaction of various components of professional activity and coping behaviour, and other factors of psychological well-being of an individual as a person, as a partner and a professional. In the course of professionalization - building up skills - the teacher also builds up stress resistance, it is one of the most striking manifestations of acme in professional development [29]. Therefore, the growth of stress resistance is associated with the success of the formation and development of a person in all spheres of his life: as a person, a partner and a professional. At the same time, personal and interpersonal stress resistance inevitably contributes to professional stress resistance.

Personal stress resistance is associated with the ability and willingness to productively and effectively transform intrapersonal difficulties and conflicts that arise when a person is faced with crises and difficult life situations that affect him as an individual. Of course, this component of the integral system of human stress resistance is closely related to others, its introduction is due only to the need to emphasize the presence of stresses that are important for a person, first of all, as for a person. This includes, for example, age-related crises, crises of personal re-identification, etc.

Interpersonal stress resistance is associated with the ability and willingness to productively and effectively transform interpersonal conflicts and correct blockages and 
deformations of relationships with other (significant) people. It is obvious that some of the significant people are, in the case of a teacher, other subjects of educational interaction. The relationship itself is the leading characteristic of an individual as a person: the selection of this aspect has only a conditional meaning. Therefore, interpersonal stress resistance as a component of a person's integral stress resistance is also difficult to separate from the other two.

Professional stress resistance involves the ability to manage oneself in professional and professionally significant situations. It is an important professional quality of a specialist in the field of education, closely related to two other components or aspects of an individual 's (trans)formation (as a person and as a partner).

Therefore, work in the field of increasing the teacher's stress resistance should be systematic and technological in nature. The preservation and strengthening of the spiritual, moral, psychological and physical health of the teacher is a necessary condition for ensuring the quality of education, as well as improving the quality of life of each subject of educational relations. Among the various methods and forms of work to prevent and overcome the syndrome of psychological burnout, professional and other deformations, didactogenies, etc., as well as to prevent and cope with injuries, difficult life situations, (di)stress, minimization of conflict relations, bullying, etc. resentment in education, are professionally, personally and interpersonally oriented interactive methods that involve (inter) active participation and inclusion in the process of psycho-prophylaxis and psychocorrection of stresses of all participants in the pedagogical process. Examples of such forms and methods of work are presented by workshops and debriefings, trainings and focus groups, etc. In the field of education and training of teachers, educational forms of work on the formation and development of stress resistance of preschool teachers can also be widely used: options for personality-oriented, contextual, problem-based or active learning, design of author's systems of professional activity with their subsequent testing and correction in pedagogical practice. In any case, used by teachers in the framework of programs of psychological and pedagogical support of their work in preschool educational institutions and at the university, as well as in the framework of their personal and interpersonal formation and development in general, methods of correction and prevention of stress and increasing stress resistance of teachers in working with children and other educated and learners, require an understanding of the essence of stress and associated options for deformities and their prevention, correction (coping) and human development. Especially productive and effective are technologies and techniques that pay attention to the development of reflection and responsibility of teachers, to strengthening a life-affirming, optimistic attitude to life, to the importance of a realistic and courageous attitude to external and internal difficulties, to designing and structuring the field of problems facing a person, to the choice of a position that is appropriate, authentic, to the person himself and allows you to build a harmonious, congruent relationship with the world.

\section{Conclusion}

Psychopedagogical ecology or ecological psychology of education is a field of ecology that studies the relationship of an individual as a system with various psychosocial phenomena, pays great attention to the correction of incorrect ones and the formation of models of behavior that are correct for an individual as an integrity (D. Arcus, W. Bronfenbrenner, J. Kagan, M.V. Osorina, V.I. Panov, N. Snidman, E.A. Sterligova) [30, 31]. A preschool educational institution is an "environment" and the task of teachers is to make this environment comfortable and safe for the "organism" (preschooler's individual). Greening the preschool environment is impossible without the formation of an effective system of environmental education and training for preschoolers. The process is the subject of study 
of pedagogical ecology (a scientific discipline, the formation of which is predetermined by the requirement of the time) ensuring the environmental safety of life at all levels of human interaction with the world around him / her. S.L. Rubinstein wrote that in a child, the development of the psyche is inextricably linked with the development of the content of human culture and the system of interpersonal relations established in this environment. D.B. Elkonin considers his social environment as a source of the child's development, which contains some ideal, exemplary forms transmitted to him by teachers and parents, guiding the child's real development [31].

Resistance to stress is a complex entity. It stabilizes and allows the development of professional competencies, human activities, including in conditions of distress. It appears as a professionally significant quality of a preschool teacher, an important criterion for his professional competence. The formation and improvement of stress resistance of teachers of preschool educational institutions occurs in the process of more or less successful solution of the tasks of professional activity, the transformation of difficulties and problems of this activity into tasks, the construction of subject-subject relations as relations of dialogue and mentoring. In the team of teachers and in the process of preparing students, procedures and classes devoted to active, joint, modeling and designing activities of prevention and coping with stresses are especially productive in solving problems that are typical and atypical for a preschool teacher. Involvement and awareness focus on development and harmonization, increasing competence and active coping, make it possible to qualitatively prepare future specialists for solving the problems of coping with various difficulties and crises, transformations and changes in educational relations.

\section{References}

1. I.V. Abaeva, A.Kh. Tsallagova, Modern technologies in education 17, 3-8 (2017)

2. M.R. Arpentieva, K.M. Gaidar, L.A. Kunakovskaya, Professional education in the modern world 10(4), 4331-4346 (2020)

3. M.R. Arpentieva, A.I. Tashcheva, S.V. Gridneva, Professional education in the modern world 10(3), 4130-4145 (2020)

4. I.Y. Aksarina, S.K. Dossayeva, A.V. Kosov, G.A. Stepanova, I.Y. Akentyeva, S.N. Brovkina, A.I. Kozhedyorov, M.R. Arpentieva, R.I. Khoteeva, K.G. Kassymova, Bulletin of the National Academy of Sciences of the Republic of Kazakhstan, 4 (380), 123-131 (2019)

5. G.K. Kasymova, G.V. Valeeva, O.P. Stepanova, O.A. Goroshchenova, R.R. Gasanova, A.A. Kulakova, P.V. Menshikov, M.R. Arpentieva, S K. Dossaeva, G.V. Garbuzova, Bulletin of the National Academy of Sciences of the Republic of Kazakhstan, 6 (382), 288-300. (2019)

6. O.Yu. Bagadaeva, Materials of the international scientific and practical conference "Information society: health, economy and law" (March 13-14 , 2019). Irkutsk: Network institute of additional professional education, 15-23 (2019)

7. O.Yu. Bagadaeva, Competence-based approach to the formation of stress resistance of teachers of preschool educational organizations (Irkutsk: Publishing house "Asprint", 2020)

8. M.G. Golubchikova, Problems of modern pedagogical education. Yalta, 62(4), 55-57 (2019)

9. M.G. Golubchikova, A.I. Akhmetova, O.M. Kolomiets, M.R. Arpentieva, IOP Conference Series: Earth and Environmental Science, 459, 052086, 1-6 (2020) https://doi.org/10.1088/1755-1315/459/5/052086 
10. G. Suyunova, M. Karimova, M. Golubchikova, M. Arpentieva, O. Kolomiets, E3S Web of Conferences, 135, 04019

11. H.H.B. Selye, Stress without distress (Moscow: MARTIS, 2018)

12. A. S. Waterman, J. of Personality and Social Psychol., 64, 678-691 (1993)

13. S. E. Hobfoll, R. J. Johnson, N. Ennis, A. P. Jackson, J. of Personality and Social Psychol. 84, 632-643 (2003)

14. O.Yu. Bagadaeva, Modern preschool education. Theory and practice 10 (72), 26-37. (2016)

15. O.Yu. Bagadaeva, M.G. Golubchikova, Pedagogical image, 4 (37), 129-141 (2017)

16. V.I. Morosanova, N.G. Kondratyuk, I.V. Gaidamashko, Moscow University Bulletin. Episode 14: Psychology, 1, 77-95 (2020)

17. S. B. Velichkovskaya, The dependence of the emergence and development of stress on the factors of professional activity of teachers: $\mathrm{PhD}$ dissertation in psychology (Moscow, 2005)

18. P. Vaclavik, How to become unhappy without outside help (Moscow: Progress, 1989)

19. Z.I. Tyumaseva, G.K. Kasymova, R.Kh. Shaimardanov, L.V. Elmendeeva, E.I. Minaeva, O.V. Gorgots, M.R. Arpentieva, A.V. Kosov, Bulletin of the National Academy of Sciences of the Republic of Kazakhstan, 4 (386), 274-283 (2020)

20. S.Yu. Vizitova, Psychological features of a teacher's resistance to stress and ways to increase it. PhD dissertation in psychology (Moscow-Yelets: Moscow Psychological and Social Institute, 2012)

21. E.V. Dubinskaya, Psychology and Pedagogy in Crimea: Ways of Development, 1, 139151 (2020)

22. A.A. Khadartsev, A.R. Tokarev, I.L. Trefilova, Bulletin of New Medical Technologies, 4, 122 (2019)

23. E.V. Barinova, Magistracy Bulletin, 1-2, 109-110 (2019)

24. A.S. Balyk, I.P. Makarenko, Society: sociology, psychology, pedagogy 11 (67), 102106 (2019)

25. T.A. Bergis, Yu.S. Krivulya, Karelian scientific journal, 9, 1 (30), 47-49 (2020)

26. R. Matušů, Lifelong Education: the XXI century, 4 (32), 90-101 (2020)

27. E.A. Zakharenko, The world of pedagogy and psychology, 11 (52), 106-115 (2020)

28. Artemieva T.V. Education and self-development, 4 (42), 79-82 (2014)

29. M.M. Kashapov, Yu.O. Ivanova, Bulletin of the Yaroslavl State University. P.G.Demidov. Humanities Series, 2 (40), 89-94 (2017)

30. V.V. Lisnichenko, N.B. Lisnichenko, Fundamentals of Pedagogical Ecology (Severodvinsk: Severodvinsk City Printing House, 2015).

31. E.A. Sterligova, Environmental psychology (Perm: Perm state nat. research university, 2012) 